\title{
Chapter 33 \\ Mechanical Strength of Poly Nanofiber Patch Under a Biaxial Tensile Loading
}

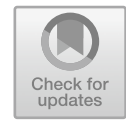

\author{
Elif Sensoy and Mahmoud Chizari
}

\begin{abstract}
Most conventional material testing apparatuses are unable to assess polynanofibers sheets in biaxial directions. This study reports the design and prototyping of a biaxial tensile apparatus which can measure the mechanical property of a poly nanofibers patch. Several samples were assessed using the designed biaxial tensile testing machine and results recorded. Function of the apparatus was validated versus convention methods and outcome confirmed that it is accurate and reliable for testing poly nanofibers patch.
\end{abstract}

Keywords Biaxial tensile testing $\cdot$ Nanofibers $\cdot$ Electrospinning $\cdot$ Poly lactic acid $\cdot$ Mechanical properties

\subsection{Introduction}

Nanofibers are defined as a material fabricated with nanometres fibres. Development and research on the nanofibers have become the interest of many industries [1]. The aim of this project is to design and develop a machine to measure the mechanical properties of poly-nanofiber sheets. This has been of interest to many different sectors including; bio medical engineering, textiles, automotive and many more. The interest in the topic has risen due to a rise in the different application of nanofibers [2]. This report will be focusing on testing the mechanical properties of poly-nanofiber patches for use of biomedical engineering and tissue engineering. Currently there isn't a very robust or reliable method of measuring the mechanical strength of poly-nanofibers which causes issues when developing nanofiber patches for uses where they will be required to have high levels of mechanical strength. The current Biaxial tensile machines available for use are not sufficient for testing poly nanofibers, as these machines have tolerances only suitable for testing conventional materials such as metals and some composites. As the demand for poly-nanofibers increase, suitable methods of testing are required, this report will show the design and development

E. Sensoy $(\varangle) \cdot$ M. Chizari

University of Hertfordshire, College Lane, Hatfield AL10 9AB, UK

e-mail: Elifsensoy1997@gmail.com 
process and the testing process of an apparatus which is suitable for testing the mechanical properties of poly-nanofibers. The design considered must be able to test specimen with a size of $30 \times 30 \mathrm{~mm}$. The measurement should be in biaxial directions and the system should be able to distribute enforced load evenly across the specimen in cross directions. The grippers holding the specimen in place, must not damage the nanofibers specimen, whilst also being able to provide enough friction to be able to hold the specimen in place and distribute the enforced load evenly. The system should be able to measure the displacement and stretch displacement of the material. It should also let the operator observe the mode of failure on the specimen. The system must be safe to use and handle. The measured data in form of force and displacement must be transferable to a computer. The results must be available for transferring into other mathematical analysis software, i.e. Microsoft excel. Furthermore, the grippers must come with sensors to consider necessary friction on the specimens.

\subsection{Design Methodology}

Once the technical design specifications were thoroughly analysed, complying design concepts were developed. Each concept was considered with conjunction to the time frame available, cost, and environmental impact. Different mechanism design options were developed and thought about, the design concepts were compared, and some elements were used in conjunction with another. The most feasible and well performing designs were taken forward. Nearly all the design concepts featured both electronic and mechanical elements. This unity provides accuracy, precision and practicality during both production and the use of the design. During design development, each design was thought about carefully, they were constantly changed to be able to improve the design even more. There were several different iterations of the same design with some elements changed, to be able to improve the overall outcome. The goal was to create a flexible design with the ability to change the parts practically during and after the design process [3]. It was important to also find a reliable method of transferring data from the Arduino software to Microsoft Excel, after thorough research it was concluded that Tera-Term would be used and integrated into the Arduino programme, so that the data yielded from the tests could be viewed in an Microsoft Excel document. The tests are to be completed 10 times to ensure that the experiment allows for accurate results to be yielded and to ensure that the test is repeatable.

The device was calibrated through Arduino programming and to ensure that the calibration done through programming software was accurate and successful, a ruler was used and attached next to the arm, to check if the measurement from the displacement mechanism made up of a small pulley mechanism. The load cells were calibrated using a 100 grams weight which was previously measured several times, then the weights were put on the load cell, to ensure that the load cells were recognising the correct values of load and force applied. This was done to ensure the repeatability of 
(a)

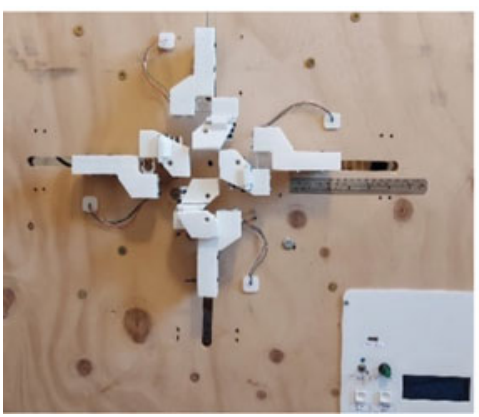

(b)

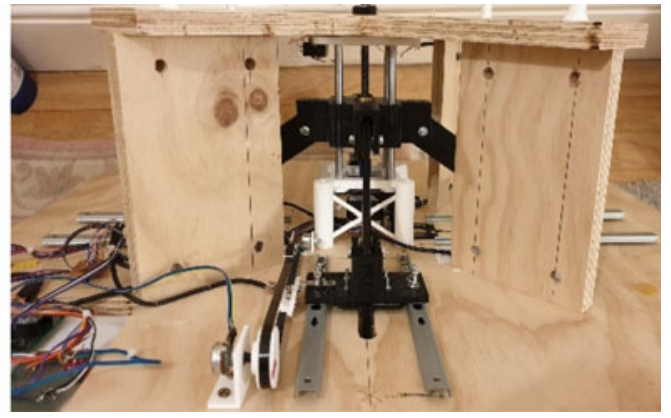

Fig. 33.1 Front view of apparatus (a), back of apparatus showing the operating mechanism

the experiment. The values measured from both the load cells and the displacement mechanism are all displayed on the LCD display on the control panel (Fig. 33.1). Following the prototyping of the machine, it was ready for a practical evaluation. To test a sample following stages were performed:

- Connect the apparatus to mains power socket and to the PC via the USB port on the control panel of the device.

- Turn on the power of the motor using the motor power switch located on the control panel, then reset the arm position and sensors by pressing load cell reset and displacement reset buttons also located on control panel.

- Ensure the specimen is ready to place and secure into the apparatus grippers. Lift the latches of each gripper and carefully place the specimen into the grippers. Close all lids and screw latches into place with the help of a screwdriver, for extra support hold the arm from the bottom to make sure the screws are in place properly.

- On the PC launch TeraTerm, once it has launched, a page called TeraTerm: New connection will come up, now select the serial option, and select the USB port used to connect the apparatus to the PC. Click OK.

- Once step 4 is complete, a window named COMX-Tera Term VT will show up on your screen. In the top, left hand corner click file, then click on log. Give the data sheet a name and add .csv to the end of the document. This will enable the data sheet to be opened as a Microsoft Excel document, once the document is named using the correct file extension click save.

- Now select file again, and under file click show dialog box, a window called TeraTerm Log will show up on the screen. Click pause so the data logging stops at that moment in time.

- Ensure that everything is secured in place and ready for testing, then click start and rotate the potentiometer located on the control panel. This will start the test and exert the load on the specimen by pulling it in all 4 directions. 
- Ensure to observe how the material tears, and once completely torn apart, go back to the PC and click pause on the TeraTerm Log window. Close the Tera Term log and COMX- Tera Term VT Windows.

- Go to the file where the data sheet saved from the previous steps, the file will automatically launch as an excel sheet.

\subsection{Test Results}

When the design and prototyping process was complete, the performance of the design had to be tested, the designs ability to test nanofibers mechanical properties, the accuracy and reliability of the results provided through the testing achieved using the device. The data yielded from testing are all within range of the average. Thus, proving the accuracy and reliability of the device. The results of the experiments complete can be seen in Figs. 33.2 and 33.3.

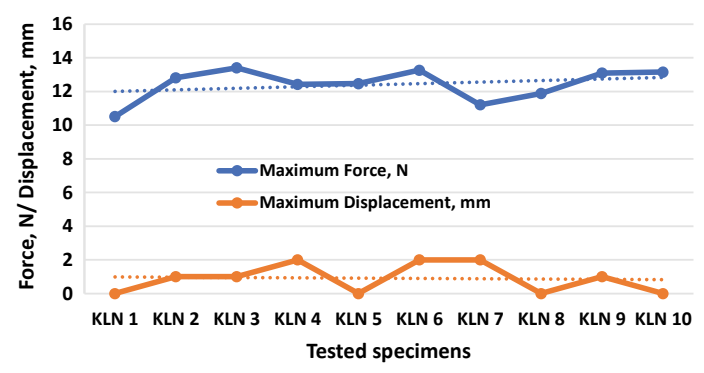

\begin{tabular}{|l|c|c|}
\hline $\begin{array}{l}\text { Testes } \\
\text { samples }\end{array}$ & $\begin{array}{l}\text { Max. } \\
\text { Force, } \mathbf{N}\end{array}$ & $\begin{array}{l}\text { Max. } \\
\text { Displacement, } \text { mm }\end{array}$ \\
\hline KLN 1 & 10.515 & 0 \\
\hline KLN 2 & 12.816 & 1 \\
\hline KLN 3 & 13.408 & 1 \\
\hline KLN 4 & 12.421 & 2 \\
\hline KLN 5 & 12.474 & 0 \\
\hline KLN 6 & 13.269 & 2 \\
\hline KLN 7 & 11.212 & 2 \\
\hline KLN 8 & 11.887 & 0 \\
\hline KLN 9 & 13.099 & 1 \\
\hline KLN 10 & 13.159 & 0 \\
\hline Avg. & 12.426 & 0.9 \\
\hline
\end{tabular}

Fig. 33.2 Graph of maximum load and displacement and table of measured load and displacement

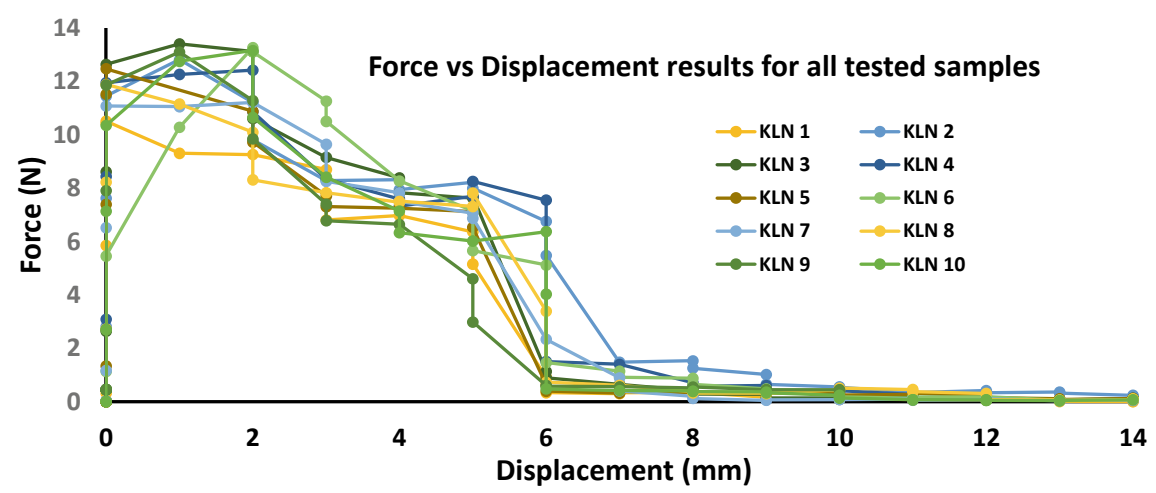

Fig. 33.3 Test results of 10 samples; Force (N) vs Displacement (mm) 


\subsection{Discussion}

The data yielded from tests completed, have illustrated the reliability and the accuracy of the device prototyped. The results produced from all 10 experiments conducted are all within the range of the average of the results yielded from the testing calculated in Fig. 33.2 as $0.9 \mathrm{~mm}$ for displacement and $12.426 \mathrm{~N}$ for maximum force. This shows that the experiment is repeatable, this also shows that the results obtained through testing and experimentation are reliable and accurate.

As can be seen in Figs. 33.2 and 33.3 some results can be classified as outliers, looking at the maximum forces in the Fig. 33.2 it can be seen that the sample, KLN 7, has a lower value than the other maximum forces yielded from the other test iterations. This could be due to different parameters such as, microtears in ply of tissue used, micro cracks due to production procedures and handling techniques e.g. fold lines. There are, of course, many parameters which could have caused inaccuracies in the results obtained, for example the homogeneity of the fibres in the Kleenex facial tissues (Kimberly-Clark Worldwide, US), and the creases caused due to the packaging of the tissues. This can also be seen from the graphs obtained through the results obtained. There are some results which can be classified as outliers, these outliers which may have been caused by the parameters discussed previously. The reason for using Kleenex tissues as test specimen is due to the ongoing adverse circumstance and unavailability of resources.

There is positive correlation between the force applied and the amount of displacement that takes place before the material deforms this can be seen in Fig. 33.3, where the materials displacement increases as the force exerted increases until the material fractures. The results which can be seen in Figs. 33.2 and 33.3 were obtained by keeping all parameters in control and steady where possible, to ensure the results yielded illustrated clearly how accurate the device is. An interesting observation made from the results obtained through testing is that the specimen undergoing testing, behaves similarly to a brittle material, meaning that the load undergone increases rapidly but no displacement is recorded, then the maximum force is reached which causes the material to fracture, then the specimen rapidly starts tearing and deforming (Fig. 33.4).

\subsection{Conclusion}

This study documents the design, development and prototyping of a biaxial tensile testing machine produced for the purposes of testing poly nanofiber patches. The design and prototype were validated through a series of comprehensive sample testing and data processing. The data yielded from testing were analysed and it was concluded that the result generated from the experiment were accurate and reliable, also that the design developed, and prototype was sufficient and performed well for the purposes of testing poly nanofibers. 
Fig. 33.4 Circuitry located behind control panel

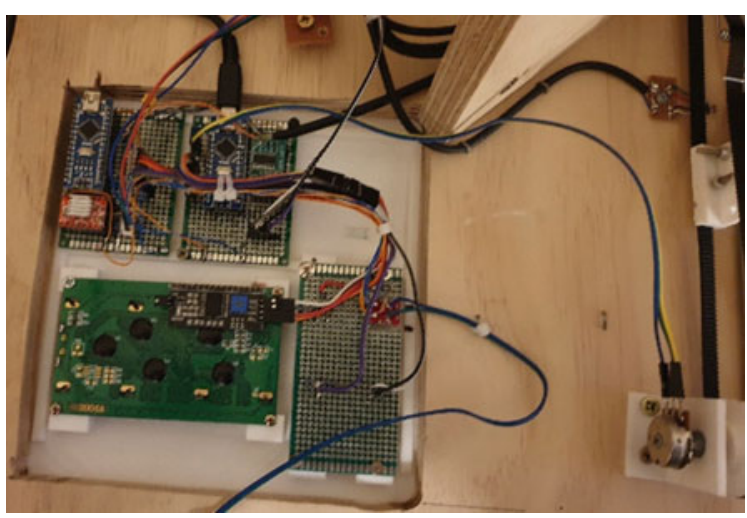

\section{References}

1. D. Alejandro Arellano Escarpita. Biaxial Tensile Strength Characterization of Textile Composite Materials. Tlalpan, México D.F., Mexico: Mechatronics Engineering Department, Instituto Tecnológico y de Estudios Superiores de Monterrey, Campus Ciudad de México, Col. Ejidos de Huipulco (2012)

2. Injection rate may effect morphology of nanofibres made by electrospinning. S. Rafiei, A. Nourani, M.H. Abedini, F. Manshae, M. Mohseni, M. Chizari. Istanbul, Turkey: 5th international conference on advances in mechanical engineering, Y1ldız Technical University, 17-19 December 2019

3. R. Casasola, N.L. Thomas, A. Trybala, S. Georgiadou, Electrospun Poly Lactic Acid Fibres: Effect of Different Solvent on Fibre Morphology and Diameter (Loughborough University, Loughborough, Leicestershire, 2015)

Open Access This chapter is licensed under the terms of the Creative Commons Attribution 4.0 International License (http://creativecommons.org/licenses/by/4.0/), which permits use, sharing, adaptation, distribution and reproduction in any medium or format, as long as you give appropriate credit to the original author(s) and the source, provide a link to the Creative Commons license and indicate if changes were made.

The images or other third party material in this chapter are included in the chapter's Creative Commons license, unless indicated otherwise in a credit line to the material. If material is not included in the chapter's Creative Commons license and your intended use is not permitted by statutory regulation or exceeds the permitted use, you will need to obtain permission directly from the copyright holder.

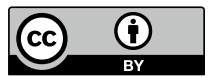

